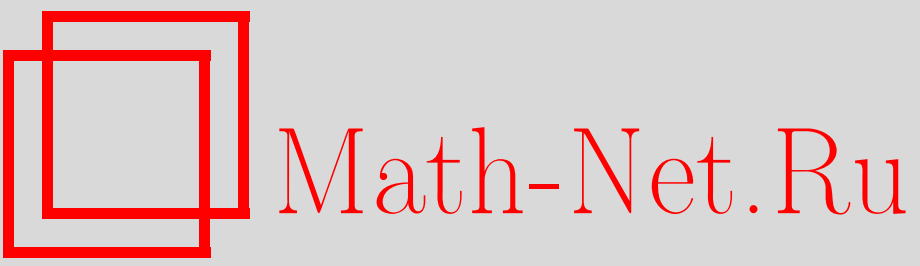

В. Р. Халилов, Нейтральный фермион с магнитным моментом во внешних электромагнитных полях, ТМФ, 2001, том 126, номер 3, 427-442

DOI: https://doi.org/10.4213/tmf439

Использование Общероссийского математического портала Math-Net.Ru подразумевает, что вы прочитали и согласны с пользовательским соглашением

http://www.mathnet.ru/rus/agreement

Параметры загрузки:

IP: 52.6 .47 .48

26 апреля 2023 г., 17:47:11 
ТЕОРЕТИЧЕСКАЯ

И МАТЕМАТИЧЕСКАЯ

ФИЗИКА

Том 126, № 3

март, 2001

(C) 2001 г.

В.Р. Халилов*

\section{НЕЙТРАЛЬНЫЙ ФЕРМИОН С МАГНИТНЫМ МОМЕНТОМ ВО ВНЕШНИХ ЭЛЕКТРОМАГНИТНЫХ ПОЛЯХ}

Взаимодействие массивного нейтрального фермиона, обладающего статическим (спиновым) магнитным дипольным моментом $\mu$, с внешним электромагнитным полем описывается уравнением Дирака-Паули. Найдены точные решения этого уравнения и соответствующий спектр энергий для внешнего магнитного поля с аксиальной симметрией и для некоторых центрально-симметричных электрических полей. Показано, что спин-орбитальное взаимодействие нейтрального фермиона с магнитным моментом определяет как характерные свойства квантовых состояний, так и спектр энергий фермиона. Установлено, что: 1) дискретный спектр энергий нейтрального фермиона зависит от проекции спина фермиона (на определенную ось квантования); 2) низший уровень энергии фермиона в рассматриваемых электрических полях и все уровни энергии (с одним определенным значением квантового числа, характеризующего проекцию спина фермиона) связанных состояний в электрическом поле напряженности $E=$ er вырожденны со счетной бесконечной кратностью; 3) спектры энергий нейтральных фермионов и антифермионов, обладающих спиновыми магнитными моментами, в центрально-симметричных электрических полях симметричны. Связанные состояния нейтрального фермиона с магнитным моментом в некоторых внешних электрических полях существуют, даже если уравнение Дирака-Паули не содержит явно члена с фермионной массой. Кроме того, в центрально-симметричных электрических полях существует счетное бесчисленное множество пар изолированных зарядово-сопряженных решений уравнения Дирака-Паули с нулевой энергией.

\section{1. ВВЕДЕНИЕ}

В релятивистской квантовой теории движение заряженной частицы со спином $1 / 2$ во внешнем электромагнитном поле описьвается уравнением Дирака, содержашим так называемое "минимальное" взаимодействие заряженного фермиона с векторным потенциалом внешнего электромагнитного поля $A_{\mu}(x)$. Паули [1], изучая лагранжианы релятивистских заряженных фермионов во внешних электромагнитных полях, указал, что добавлением к лагранжиану дополнительных членов, содержащих явно напряженности внешнего поля $F_{\mu \nu}(x)$, можно описать дополнительные (аномальные) магнитные моменты (AMM) заряженных фермионов. Заметим, что в работе [1] рассматривались лишь

\footnotetext{
* Московский государственный университет, Москва, Россия. E-mail: khalilov@thc.phys.msu.su
} 
дополнительные взаимодействия, не изменяющие симметрию исходного лагранжиана, и, в частности, АММ нейтрона можно описать введением в лагранжиан дополнительного взаимодействия указанного типа. Если инвариантность взаимодействий элементарных частиц по отношению к обрашению времени (или $C P$-инвариантность, как в распаде $K_{2}^{0} \rightarrow \pi^{+} \pi^{-}$) не является универсальным принципом природы, то фермионы могут обладать электрическими дипольными моментами (ЭДМ) [2]. ЭДМ заряженного фермиона можно учесть феноменологически, вводя в лагранжиан дополнительный калибровочно-инвариантный, но не инвариантный относительно $T$ - и $P$-отражений, член взаимодействия [3].

Учет дополнительных членов, связанных с АММ и ЭДМ заряженного фермиона, существенно усложняет структуру и нахождение точных решений полученного из такого лагранжиана обобшенного уравнения Дирака в присутствии внешнего электромагнитного поля. Точные решения обобщенного уравнения Дирака для заряженного фермиона, обладающего АММ и ЭДМ, известны лишь в некоторых специальных конфигурациях электромагнитных полей: в поле плоской электромагнитной волны [4], [5], в произвольном постоянном электромагнитном поле [6] и для одного класса постоянных неоднородных электрических полей [7], [8].

Точные решения обобшенного уравнения Дирака для нейтрального фермиона с АММ (это уравнение называют уравнением Дирака-Паули) в постоянном неоднородном магнитном поле специальной конфигурации и в некоторых неоднородных электростатических полях обсуждались в работах [9] и [10], соответственно.

В последнее время снова возник интерес к этим задачам. На наш взгляд, этот интерес связан с рядом замечательных событий, среди которых можно выделить следующие: открытие нейтронных звезд с гигантскими индукциями магнитных полей и сверхвысокими плотностями нейтронов и протонов в центральных областях этих звезд; создание квантово-полевых теоретических моделей, предсказывающих возможность существования АММ и даже ЭДМ у массивного дираковского нейтрино (индуцированных радиационными поправками во внешнем электромагнитном поле); предсказание [11] и открытие [12] эффекта Ааронова-Кашера, прямо указываюшего на сушествование и важную роль “неминимального" взаимодействия. Отметим, что задача о рассеянии нейтронов на ядрах, обусловленном (прямым) “неминимальным" взаимодействием АММ нейтрона с внешним электростатическим полем ядра, впервые рассматривалась Швингером [13].

В настоящей работе мы изучим решения уравнения Дирака-Паули для массивного нейтрального фермиона, обладающего статическим (спиновым) магнитным дипольным моментом, во внешнем аксиально-симметричном магнитном поле и в некоторых центрально-симметричных электрических полях. Задача может представлять интерес для физики элементарных частищ и астрофизики.

Мы используем систему единиц, в которой $c=\hbar=1$, и выбрали так называемое стандартное представление матриц Дирака [14]: $\gamma^{0}=\gamma_{0} \equiv \beta, \gamma^{5}=\gamma_{5}=i \gamma_{0} \gamma_{1} \gamma_{2} \gamma_{3}, \boldsymbol{\alpha}=$ $\gamma^{0} \gamma, \sigma=\gamma^{5} \gamma \gamma^{0}$ 


\section{2. ОБОБШЕННОЕ УРАВНЕНИЕ ДИРАКА}

Лагранжиан фермиона с массой $m$, зарядом $е$ и АММ $\mu$, взаимодействуюшего с внешним электромагнитным полем, был предложен Паули [1] в виде

$$
L=\bar{\Psi}\left[\gamma^{\mu}\left(i \partial_{\mu}-e A_{\mu}\right)-m\right] \Psi-\frac{1}{2} \mu \bar{\Psi} \sigma^{\mu \nu} F_{\mu \nu} \Psi
$$

где $\gamma^{\mu}=\left(\gamma^{0}, \gamma\right)$ - матришы Дирака, удовлетворяюшие антикоммутационным соотношениям $\left\{\gamma^{\mu}, \gamma^{\nu}\right\}=2 g^{\mu \nu}$ с $g^{\mu \nu}=g_{\mu \nu}=\operatorname{diag}(1,-1,-1,-1), \sigma^{\mu \nu}=i\left[\gamma^{\mu}, \gamma^{\nu}\right] / 2, A_{\mu}-$ 4 -потенциал внешнего электромагнитного поля, $F_{\mu \nu}=\partial_{\mu} A_{\nu}-\partial_{\nu} A_{\mu}$ - тензор внешнего электромагнитного поля.

Обобщенное уравнение Дирака для массивного заряженного фермиона с АMМ, взаимодействуюшего с внешним электромагнитным полем, имеет вид

$$
\left(\gamma^{\mu} P_{\mu}-\frac{1}{2} \mu \sigma^{\mu \nu} F_{\mu \nu}-m\right) \Psi=0
$$

где $P_{\mu}=i \partial_{\mu}-e A_{\mu}$ - оператор обобшенного импульса заряженного фермиона.

Нетрудно показать, что

$$
\frac{1}{2} \sigma^{\mu \nu} F_{\mu \nu}=i \boldsymbol{\alpha} \cdot \mathbf{E}-\boldsymbol{\sigma} \cdot \mathbf{B}
$$

где $\mathbf{E}$ и $\mathbf{B}$ - напряженности электрического и магнитного полей, соответственно.

Уравнение Дирака для массивного нейтрального фермиона с АММ $\mu$ получают из уравнения (2), полагая в нем $e=0$. С учетом (3) уравнение Дирака-Паули (2) (c $e=0)$ можно записать в виде уравнения Шредингера

$$
i \frac{\partial \Psi}{\partial t}=\mathcal{H} \Psi
$$

с гамильтонианом

$$
\mathcal{H}=\boldsymbol{\alpha} \cdot \mathbf{p}-\mu(-i \boldsymbol{\gamma} \cdot \mathbf{E}+\beta \boldsymbol{\sigma} \cdot \mathbf{B})+\beta m .
$$

Сначала мы найдем точные стационарные решения одночастичного уравнения Дирака-Паули во внешних статических электромагнитных полях с "положительными" частотами (в области энергий $\mathcal{E}>0$ ), а затем обсудим решения уравнения Дирака-Паули с "отрицательными" частотами и область спектра с $\mathcal{E}<0$. Весь спектр энергий с $\mathcal{E}>0$ и $\mathcal{E}<0$ необходимо знать, для того чтобы понять, возможно ли пересечение уровней энергии, принадлежаших в отсутствие поля областям $\mathcal{E}>0$ и $\mathcal{E}<0$. Кроме того, все решения уравнения Дирака-Паули определенным образом входят в схему вторичного квантования [15] и поэтому необходимы для определения основного состояния (вакуума) вторично квантованной теории. 


\section{3. ФЕРМИОН В АКСИАЛЬНО-СИММЕТРИЧНОМ МАГНИТНОМ ПОЛЕ}

Рассмотрим движение нейтрального фермиона во внешнем аксиально-симметричном магнитном поле, вектор напряженности которого в цилиндрических координатах $r, \varphi, z$ имеет вид

$$
\mathbf{B}=\left(0,0, \frac{a}{r}+b\right) .
$$

Решение уравнения Дирака-Паули во внешнем поле (6) ишем в виде

$$
\Psi(t, \mathbf{r})=e^{-i \mathcal{E} t} \psi(\mathbf{r})
$$

где $\mathcal{E}$ - энергия фермиона, а $\psi(\mathbf{r})$ - не зависящий от времени биспинор. Спектр энергий фермиона определяется из стационарного уравнения Дирака-Паули

$$
\mathcal{H} \psi(\mathbf{r})=\mathcal{E} \psi(\mathbf{r})
$$

где гамильтониан $\mathcal{H}$ определяется формулой (5) с подстановкой (6).

Нетрудно показать, что волновая функция $\Psi$ в поле $(6)$ является также собственной функцией следующих операторов (интегралов движения): проекции импульса на направление поля (ось $z)$

$$
-i \frac{\partial \Psi}{\partial z}=p \Psi
$$

проекции полного момента на это направление

$$
J_{z} \Psi=\left(-i \partial_{\varphi}+\frac{\sigma_{3}}{2}\right) \Psi=\left(l+\frac{1}{2}\right) \Psi
$$

и спинового оператора $[9]$

$$
S \Psi=s \Psi, \quad S=m \sigma_{3}-i[\gamma \cdot \mathbf{p}]_{3}-\mu B,
$$

описывающего проекцию спина фермиона на направление магнитного поля. Эти операторы коммутируют с гамильтонианом $\mathcal{H}$, а их собственные значения $p$, $l+1 / 2$ и $s$ нумеруют решения уравнения Дирака-Паули.

С учетом этих требований биспинор $\psi(\mathbf{r})$ можно записать в виде

$$
\begin{gathered}
\psi(\mathbf{r})=\frac{\exp (i p z+i l \varphi)}{\sqrt{2 \pi L}}\left(\begin{array}{c}
\phi \\
\chi
\end{array}\right), \\
\phi(r, \varphi)=\left(\begin{array}{c}
c_{1} f(r) \\
i c_{2} g(r) e^{i \varphi}
\end{array}\right), \quad \chi(r, \varphi)=\left(\begin{array}{c}
-c_{2} f(r) \\
i c_{1} g(r) e^{i \varphi}
\end{array}\right),
\end{gathered}
$$

причем функции $f$ и $g$ удовлетворяют уравнениям

$$
\begin{array}{r}
\frac{d f}{d r}-\frac{l}{r} f+\left(s+m-\frac{\mu a}{r}-\mu b\right) g=0, \\
\frac{d g}{d r}+\frac{l+1}{r} g-\left(s-m-\frac{\mu a}{r}-\mu b\right) f=0,
\end{array}
$$


коэффициенты $c_{k}$ имеют вид

$$
c_{1}=\sqrt{\frac{1+\frac{s}{\mathcal{E}}}{2}}, \quad c_{2}=-\sqrt{\frac{1-\frac{s}{\mathcal{E}}}{2}}
$$

и выполняется соотношение

$$
\mathcal{E}=\sqrt{p^{2}+s^{2}} .
$$

Обозначим $s-\mu b=\zeta E$, где значения $\zeta= \pm 1$, очевидно, соответствуют двум возможным ориентациям спина фермиона.

Система уравнений (13) совпадает с системой уравнений в задаче о движении релятивистского заряженного фермиона во внешнем кулоновском поле в $2+1$ измерениях. Последняя задача была решена в [16]. Используя результаты работ [16], нетрудно показать, что дискретный спектр для $s$ или $E(E<m)$ возможен при $-\mu a \equiv \alpha>0$ только для $\zeta=-1$, а при $\alpha<0$ только для $\zeta=1$. Подчеркнем, что спектр энергий нейтрального фермиона в поле (6) определяется формулой (15) и является квазинепрерывным и в области $E<m$.

Функции $f(r)$ и $g(r)$ имеют вид [16]

$$
\begin{aligned}
& f=\sqrt{m+E} e^{-\frac{\rho}{2}} \rho^{\gamma-1}\left(Q_{1}+Q_{2}\right), \\
& g=\sqrt{m-E} e^{-\frac{\rho}{2}} \rho^{\gamma-1}\left(Q_{1}-Q_{2}\right),
\end{aligned}
$$

где введены обозначения

$$
\rho=2 \lambda r, \quad \lambda=\sqrt{m^{2}-E^{2}}, \quad \gamma=\frac{1}{2}+\sqrt{\left(l+\frac{1}{2}\right)^{2}-\alpha^{2}},
$$

a $\gamma$ определяет асимптотическое поведение волновых функций при малых $r$.

Решение, конечное при $\rho=0$, выражается через вырожденную гипергеометрическую функцию $F(a, b ; z)$ следуюшим образом:

$$
\begin{aligned}
& Q_{1}=A F\left(\gamma-\frac{1}{2}-\left(\frac{\alpha E}{\lambda}\right), 2 \gamma ; \rho\right), \\
& Q_{2}=D F\left(\gamma+\frac{1}{2}-\left(\frac{\alpha E}{\lambda}\right), 2 \gamma ; \rho\right),
\end{aligned}
$$

причем связь между постоянными $A$ и $D$ определяется соотношением

$$
D=\frac{\gamma-\frac{1}{2}-\frac{\alpha E}{\lambda}}{l+\frac{1}{2}+\frac{\alpha m}{\lambda}} A
$$

Остаюшийся неопределенным обший нормировочный коэффишиент $A$ можно найти из условия

$$
\int_{0}^{\infty}\left(f^{2}+g^{2}\right) r d r=1
$$


Заметим, что коэффициент $A$ проще найти, использовав асимптотический вид функций при $r \rightarrow \infty$. Мы находить $A$ не будем.

Собственные значения энергии определяются из уравнения

$$
\gamma-\frac{1}{2}-\frac{\alpha E}{\lambda}=-n,
$$

причем нетрудно показать, что допустимы следующие значения радиального квантового числа $n: 0,1,2, \ldots$ при $l \geqslant 0$ и $1,2,3, \ldots$ при $l<0$. Тогда

$$
E_{n, l}=m\left[1+\frac{\alpha^{2}}{\left(n+\sqrt{\left(l+\frac{1}{2}\right)^{2}-\alpha^{2}}\right)^{2}}\right]^{-\frac{1}{2}},
$$

а спектр энергий нейтрального фермиона в аксиально-симметричном магнитном поле (6) имеет вид

$$
\mathcal{E}=\sqrt{p^{2}+\left(E_{n, l}-\mu b\right)^{2}} .
$$

Волновые функции непрерывного спектра $(E>m)$ можно получить из функций дискретного спектра заменами

$$
\begin{gathered}
\sqrt{E+m} \rightarrow \sqrt{E+\zeta m}, \quad \sqrt{m-E} \rightarrow-i \sqrt{E-\zeta m} \\
\lambda \rightarrow-i k, \quad-n \rightarrow \gamma-\frac{i \alpha E}{k},
\end{gathered}
$$

и, разумеется, заново должна быть произведена нормировка функций. Мы не будем приводить здесь волновые функции состояний непрерывного спектра.

Итак, главная особенность состояний нейтрального фермиона с АММ $\mu$ в магнитном поле (6) состоит в том, что квазидискретньй спектр энергий существует в зависимости от знака $\mu$ только при одном значении проекции спина фермиона. Отметим также, что магнитное поле вида (6) можно создать только с помошью внешних токов, текуших во всем пространстве, так как $\operatorname{rot} \mathbf{B} \neq 0$.

\section{4. ФЕРМИОН В ЦЕНТРАЛЬНО-СИММЕТРИЧНЫХ ЭЛЕКТРИЧЕСКИХ ПОЛЯХ}

При нахождении решений уравнения Дирака-Паули для массивного нейтрального фермиона с аномальным (спиновым) магнитным моментом $\mu$ в электрических полях, обладаюших центральной симметрией, используем сферические координаты $(r, \theta, \phi)$. Вектор напряженности внешнего электрического поля зададим в виде

$$
\mathbf{E}=E(r) \mathbf{e}_{r},
$$

где $\mathbf{e}_{r}-$ единичный вектор в радиальном направлении. 
Решение уравнения Дирака-Паули ишем в виде (7). Тогда спектр энергий фермиона определяется из стационарного уравнения Дирака-Паули (8), а гамильтониан Дирака-Паули для поля (25) имеет вид

$$
\mathcal{H}=\boldsymbol{\alpha} \cdot \mathbf{p}+i \mu \boldsymbol{\gamma} \cdot \mathbf{E}+\beta m
$$

Можно показать, что в поле (25) интегралами движения являются операторы полного момента частишы $\mathbf{J}=\mathbf{L}+\mathbf{S}$, где $\mathbf{S}=\sigma / 2$, одна из проекций полного момента (на ось $z)$, а также спиновый оператор $N_{0}=\beta(\boldsymbol{\sigma} \cdot \mathbf{L}+1)$, введенный Дираком [17]. Нелишне отметить, что оператор $N_{0}=N^{0}$ является временной компонентой спинового векторного оператора [18]. Состояния рассматриваемого нейтрального фермиона в центрально-симметричных электрических полях можно выбрать как собственные состояния гамильтониана (26) и операторов $\mathbf{J}^{2}, J_{z}$ и $N_{0}$ с собственными значениями $(l+1 / 2)(l+3 / 2)$, $m+1 / 2$ и $s(l+1)$, соответственно, где для характеристики двух возможных ориентаций спина фермиона вдоль вектора момента $\mathbf{J}$ введено квантовое число $s= \pm 1$.

Волновую функцию стационарного состояния $\psi$ (в стандартном представлении матриц Дирака) ишем в виде

$$
\psi(\mathbf{r})=\left(\begin{array}{l}
\varphi \\
\chi
\end{array}\right),
$$

где $\varphi$ и $\chi$ - двухкомпонентные спиноры. Тогда стационарное уравнение Дирака-Паули принимает вид

$$
\begin{aligned}
& \boldsymbol{\sigma} \cdot\left(\mathbf{p}-i \mu E \mathbf{e}_{r}\right) \varphi=(\mathcal{E}+m) \chi, \\
& \boldsymbol{\sigma} \cdot\left(\mathbf{p}+i \mu E \mathbf{e}_{r}\right) \chi=(\mathcal{E}-m) \varphi,
\end{aligned}
$$

где $\boldsymbol{\sigma}$ - матрицы Паули.

Определим два двухкомпонентных спинора [10], [18], [19]

$$
\begin{gathered}
u_{l m}^{+}(\theta, \phi)=\left(\begin{array}{c}
\sqrt{\frac{l+m+1}{2 l+1}} Y_{l, m}(\theta, \phi) \\
\sqrt{\frac{l-m}{2 l+1}} Y_{l, m+1}(\theta, \phi)
\end{array}\right), \quad u_{l m}^{-}(\theta, \phi)=\left(\begin{array}{c}
\sqrt{\frac{l-m+1}{2 l+3}} Y_{l+1, m}(\theta, \phi) \\
-\sqrt{\frac{l+m+2}{2 l+3}} Y_{l+1, m+1}(\theta, \phi)
\end{array}\right), \\
l=0,1,2, \ldots, \quad m=-(l+1),-l, \ldots, l .
\end{gathered}
$$

Здесь $Y_{l m}(\theta, \phi)$ - шаровые функции [15] (сферические гармоники [20]). Обратим внимание, что спиноры $u^{+}, u^{-}$являются обшими собственными функциями операторов $\mathbf{J}^{2}$, $J_{z}, \mathbf{L}^{2}$ с собственными значениями

$$
\left(l+\frac{1}{2}\right)\left(l+\frac{3}{2}\right), m+\frac{1}{2}, l(l+1)
$$

и

$$
\left(l+\frac{1}{2}\right)\left(l+\frac{3}{2}\right), m+\frac{1}{2},(l+1)(l+2),
$$

соответственно.

4 Теоретическая и математическая физика, т. 126, № 3, 2001 г. 
Биспинор (27) представим в виде

$$
\begin{aligned}
\psi(\mathbf{r}) & =\left(\begin{array}{c}
\varphi \\
\chi
\end{array}\right)=\frac{1+s}{2}\left(\begin{array}{c}
\varphi^{+} \\
\chi^{+}
\end{array}\right)+\frac{1-s}{2}\left(\begin{array}{c}
\varphi^{-} \\
\chi^{-}
\end{array}\right)= \\
& =\frac{1+s}{2 r}\left(\begin{array}{c}
f_{s}(r) u_{l m}^{+}(\theta, \phi) \\
i g_{s}(r) u_{l m}^{-}(\theta, \phi)
\end{array}\right)+\frac{1-s}{2 r}\left(\begin{array}{c}
f_{s}(r) u_{l m}^{-}(\theta, \phi) \\
i g_{s}(r) u_{l m}^{+}(\theta, \phi)
\end{array}\right) .
\end{aligned}
$$

Подставляя (29) в (28), после несложных преобразований приходим к следующей системе обыкновенных дифференциальных уравнений для функций $f_{s}$ и $g_{s}$, характеризующих радиальное движение:

$$
\begin{aligned}
& \left(\frac{d}{d r}+s \frac{l+1}{r}+\mu E\right) f_{s}+(\mathcal{E}+m) g_{s}=0 \\
& \left(\frac{d}{d r}-s \frac{l+1}{r}-\mu E\right) g_{s}-(\mathcal{E}-m) f_{s}=0 .
\end{aligned}
$$

Напомним, что квантовое число $s$ принимает значения \pm 1 и характеризует ориентацию проекции спина фермиона вдоль вектора момента $\mathbf{J}$. Радиальные функции $f_{s}$ и $g_{s}$ нормированы условием

$$
\int_{0}^{\infty}\left(\left|f_{s}^{2}\right|+\left|g_{s}^{2}\right|\right) d r=1
$$

Заметим, что при заменах $\mathcal{E} \rightarrow-\mathcal{E}, \mu \rightarrow-\mu$ уравнение $(30)$ для $f_{-1}$ переходит в $(30)$ для $g_{1}$, а уравнение (30) для $f_{1}-$ в $(30)$ для $g_{-1}$. Такими же свойствами симметрии обладает система уравнений (13) для нейтрального фермиона с АММ в магнитном поле (6). Эти свойства позволяют по найденным решениям с "положительными" частотами $(\mathcal{E}>0)$ построить решения с "отрицательными” частотами $(\mathcal{E}<0)$.

Исключая из системы уравнений $(30)$ функцию $f_{s}$ или $g_{s}$, получим следующие независимые уравнения второго порядка

$$
\begin{aligned}
{\left[\frac{d^{2}}{d r^{2}}+\right.} & \mathcal{E}^{2}-m^{2}+\mu\left(-2 s E \frac{l+1}{r} \pm \frac{d E}{d r}\right)- \\
& \left.-\frac{(l+1)(l+1 \pm s)}{r^{2}}-(\mu E)^{2}\right]\left(\begin{array}{l}
f_{s}(r) \\
g_{s}(r)
\end{array}\right)=0
\end{aligned}
$$

где верхний знак относится к уравнениям для функций $f_{s}(r)$, а нижний знак - для $g_{s}(r)$. Уравнения (32) для функций $f_{s}$ и $g_{s}$ имеют вид радиального уравнения Шредингера в центральном поле с эффективными потенциалами

$$
U_{ \pm}=2 \mu E s \frac{l+1}{r} \mp \mu \frac{d E}{d r}+\frac{(l+1)(l+1 \pm s)}{r^{2}}+(\mu E)^{2} .
$$

Первый член в (33) характеризует спин-орбитальное взаимодействие массивного нейтрального фермиона, обладаюшего АММ, с электрическим полем. Мы увидим, что влияние этого члена на свойства состояний и спектр энергий нейтрального фермиона 
оказывается весьма сушественным. Далее, уже из вида (33) следует, что спектр энергий частиц лежит в области энергий $\mathcal{E} \geqslant m$. Действительно, если, например, в некоторой области $r U_{+}(r)>0$, то в той же области $U_{-}(r)<0$, и наоборот, если $U_{+}(r)<0$, то $U_{-}(r)>0$. Отсюда следует, что $\mathcal{E}^{2}-m^{2} \geqslant 0$, и, значит, низший (положительный) уровень энергии $\mathcal{E} \geqslant m$. Точные решения системы (30) можно получить для некоторых конкретных функций $E(r)$. Для определенности фиксируем знак АММ фермиона, полагая $\mu>0$; АММ антифермиона равен по величине, но противоположен по знаку, так что $\mu<0$.

4.1. Радиально-постоянное поле $E(r)=a$. Рассмотрим точные решения системы (30) в радиально-постоянном электрическом поле с $E(r)=a>0$ (случай $a<0$ заменой переменных в (30) сводится к случаю $a>0)$. Как видно из (32), все связанные состояния в этом поле сушествуют лишь при $s=-1$, причем спектр энергий связанных состояний лежит в области $\sqrt{m^{2}+(\mu a)^{2}}>\mathcal{E} \geqslant m$. Все состояния с $s=1$ принадлежат непрерывному спектру энергий. Для этих состояний $\mathcal{E}>\sqrt{m^{2}+(\mu a)^{2}}$.

Решения уравнений (32) с $s=-1$ удобно выразить через функции Уиттекера [21]:

$$
f_{-1}(r)=A W_{\beta, \gamma}(2 \lambda r), \quad g_{-1}(r)=B W_{\beta, \gamma+1}(2 \lambda r),
$$

где

$$
\beta=\frac{\mu a(l+1)}{\lambda}, \quad \lambda=\sqrt{m^{2}+(\mu a)^{2}-\mathcal{E}^{2}}, \quad \gamma=l+\frac{1}{2} .
$$

Связь между постоянными $A$ и $B$ можно найти из любого из уравнений (30), используя рекуррентные соотношения для функций Уиттекера [22]

$$
(2 \mu+1) \frac{d W_{\nu, \mu}(z)}{d z}-\left[\frac{(2 \mu+1)^{2}}{2 z}-\nu\right] W_{\nu, \mu}(z)=\left(\nu-\mu-\frac{1}{2}\right) W_{\nu, \mu+1}(z) .
$$

В результате найдем $A(\lambda-\mu a)=B(\mathcal{E}+m)$. Заметим, что в низшем связанном состоянии $(\mathcal{E}=m) \lambda=\mu a$ и, следовательно, $B=0$. Обе функции Уиттекера в (34) должны сводиться к полиномам, что имеет место при вьполнении условий

$$
\frac{1}{2}+\gamma-\beta=-n
$$

для функции $f_{-1}(r)$ и

$$
\frac{1}{2}+\gamma+1-\beta=-n
$$

для функции $g_{-1}(r)$.

Если $n=1,2, \ldots$, то обе функции Уиттекера сводятся к полиномам, если же $n=0$, то к полиному сводится лишш функция $f_{-1}(r)$. Но равенство $n=0$ означает, что $\mathcal{E}=m$, $\lambda=\mu a$, а в этом случае, как было показано выше, коэффициент $B=0$, так что $g_{-1}(r)=0$. Таким образом, из определения (36) найдем выражение для дискретного 
спектра энергий с учетом его явной зависимости от знака проекции спина нейтрального фермиона:

$$
\mathcal{E}_{n l s}=\left[m^{2}+(\mu a)^{2} \frac{(n+l+1)^{2}-(l+1)^{2}}{(n+l+1)^{2}}\right]^{\frac{1}{2}} \frac{1-s}{2}, \quad n=0,1,2, \ldots
$$

Здесь $n$ имеет смысл радиального квантового числа. Видно, что низший уровень энергии $\mathcal{E}_{0 l}=m$ не зависит от $l$, т.е. уровень энергии с $n=0$ вырожден с (счетно) бесконечной кратностью [19].

Состояния непрерывного спектра $\left(\mathcal{E}>\sqrt{m^{2}+(\mu a)^{2}}\right)$ можно получить из функций дискретного спектра с помощью замен типа (24). Мы их здесь приводить не будем, подчеркнем только, что при выбранном знаке АММ фермиона $\mu>0$ и электрического поля $a>0$ проекция спина фермиона на вектор момента $\mathbf{J}$ может принимать только одно значение $s=1$. Итак, состояния нейтрального фермиона с АММ $\mu$ в радиально-постоянном электрическом поле имеют две главные особенности: основной уровень энергии дискретного спектра вырожден с (счетно) бесконечной кратностью; как дискретный, так и непрерывный спектры энергий сушествуют в зависимости от знака $\mu$ только для одного значения проекции спина фермиона.

4.2. Центрально-симметричные поля $E(r)=e r$ и $E(r)=a / r$. Найдем точные решения системы (30) с "положительными" частотами в электрическом поле $E(r)=e r$ с $e>0$ (полагаем также, что $\mu>0$ ). Из (32) нетрудно установить, что в поле $E(r)=$ $e r>0$ связанные состояния существуют для двух значений квантового числа $s= \pm 1$. Решение задачи полностью аналогично решению известной задачи нерелятивистской квантовой механики - уравнения Шредингера для осцилляторного потенциала. Вьполнив простые и стандартные процедуры в уравнениях (32), нетрудно показать, что функции $f_{s}(r)$ и $g_{s}(r)$, конечные при $r \rightarrow 0$, можно представить в виде

$$
\begin{aligned}
& f_{s}(r)=A r^{l+1} e^{-\frac{|\mu e| r^{2}}{2}} F\left(a, b ;|\mu e| r^{2}\right), \\
& g_{s}(r)=B r^{l+2} e^{-\frac{|\mu e| r^{2}}{2}} F\left(a+1, b+1 ;|\mu e| r^{2}\right),
\end{aligned}
$$

где введены обозначения

$$
a=\frac{1}{2}\left(l+\frac{4+s}{2}-d\right), \quad d=\frac{\mathcal{E}^{2}-m^{2}-s \mu e(2 l+2-s)}{2|\mu e|}, \quad b=l+\frac{3}{2},
$$

a $F(a, b ; z)$ - вырожденная гипергеометрическая функция.

Дискретный спектр энергий фермиона определяется из условия

$$
a=-n,
$$

тогда

$$
d=2 n+l+\frac{4+s}{2}
$$


откуда получим

$$
\mathcal{E}_{n l s}=\sqrt{m^{2}+|\mu e|[4 n+(2 l+3)(1+s)]},
$$

где $n$ имеет смысл радиального квантового числа. В полученном выражении уровни энергии (42) явно зависят от ориентации спина нейтрального фермиона. Видим также, что все уровни энергии с $s=-1$ не зависят от $l$, т.е. все эти уровни энергии вырожденны с (счетно) бесконечной кратностью. Связь между постоянными $A$ и $B$ можно найти из любого из уравнений (30), используя рекуррентные соотношения для вырожденных гипергеометрических функций. В результате найдем $B(2 l+3)=-A(s \mathcal{E}+m)$. Отметим, что в низшем связанном состоянии с $s=-1, n=0(\mathcal{E}=m) B=0$.

Заметим также, что все найденные выше решения можно записать в терминах одной из специальных функций - вырожденной гипергеометрической функции $F(a, b ; z)$, функции Уиттекера $W_{\beta, \gamma}(z)$ или полинома Лагерра $L_{r}^{k}(z)$, используя соотношения между этими функциями [21]

$$
\begin{aligned}
W_{l+\mu+\frac{1}{2}, \mu}(z) & =(-1)^{l} z^{\mu+\frac{1}{2}} e^{-\frac{z}{2}}(2 \mu+1)(2 \mu+2) \ldots(2 \mu+l) F(-l, 2 \mu+1 ; z)= \\
& =(-1)^{l} z^{\mu+\frac{1}{2}} e^{-\frac{z}{2}} L_{l}^{2 \mu}(z),
\end{aligned}
$$

справедливые при условии, что $l+1$ - натуральное число.

В электрическом поле $E(r)=a / r$ с $a>0$ (полагаем, как и выше, $\mu>0$ ) сушествуют только состояния непрерывного спектра. Точные решения системы (30) с “положительными" частотами выражаются через функции Бесселя $J_{\nu}(z)$ и функции Неймана $N_{\nu}(z)$ индекса

$$
\nu=\mu a-s(l+1) \mp \frac{1}{2}
$$

следующим образом:

$$
f_{s}=\sqrt{r}\left(A J_{|\nu|}(\kappa r)+C N_{|\nu|}(\kappa r)\right), \quad g_{s}=\sqrt{r}\left(B J_{|\nu|}(\kappa r)+D N_{|\nu|}(\kappa r)\right),
$$

где

$$
\kappa=\sqrt{\mathcal{E}^{2}-m^{2}}
$$

и верхний знак в выражении для $\nu$ относится к функции $f_{s}$, а нижний знак - к функции $g_{s}$. Для конечности функций при $r=0$ необходимо, чтобы $C=D=0$. Отметим, что плоская конфигурация электрического поля $E=a / r$ соответствует полю, в котором наблюдался эффект Ааронова-Кашера, но конфигурация поля в эксперименте [12] скорее соответствовала аксиальной (а не центральной) симметрии.

4.3. Спектр энергий античастиц. Отрицательные собственные значения одночастичного гамильтониана Дирака-Паули нужно знать (уже в рамках одночастичной задачи) в случае пересечения уровней энергии, принадлежащих в отсутствие поля ветвям $\mathcal{E} \geqslant m$ и $\mathcal{E} \leqslant-m$. Решения уравнения Дирака-Паули с "отрицательными" частотами влияют на определение вакуумного состояния вторично квантованной теории. Поэтому обсудим кратко спектр “отрицательных" энергий одночастичного уравнения Дирака-Паули. 
Обозначим нормированные (соответствуюшим образом) собственные функции уравнения Дирака-Паули, относяшиеся к положительным $\left(\mathcal{E}_{n}^{+}>0\right)$ и отрищательным $\left(\mathcal{E}_{n}^{-}<0\right)$ собственным значениям одночастичного гамильтониана Дирака-Паули во внешнем поле, $\psi_{n}^{+}(x)$ и $\psi_{n}^{-}(x)$, соответственно. Отрицательно-частотные решения уравнения Дирака-Паули, описываюшие античастицу, можно получить из найденных выше положительно-частотных собственных функций с помощью преобразования зарядового сопряжения. Этим преобразованием решение уравнения Дирака-Паули с положительной энергией и проекцией спина $s$ переводится в решение с отрицательной энергией и проекцией спина $-s$. Для получения же спектра “отрицательных" энергий в найденных выше формулах (38) и (42) нужно просто заменить $s$ на $-s$. Дискретные уровни энергии, принадлежащие положительно- и отрицательно-частотным состояниям в электрическом поле, имеют вид

$$
\begin{aligned}
& \mathcal{E}_{n l s}= \pm\left[m^{2}+(\mu a)^{2} \frac{(n+l+1)^{2}-(l+1)^{2}}{(n+l+1)^{2}}\right]^{\frac{1}{2}} \frac{1 \pm s}{2}, \\
& \mathcal{E}_{n l s}= \pm \sqrt{m^{2}+|\mu e|[4 n+(2 l+3)(1 \pm s)]}, \quad n=0,1,2, \ldots
\end{aligned}
$$

Спектры и отрицательно-частотные решения уравнения Дирака-Паули в присутствии магнитного поля можно найти точно так же.

Спектры “положительных" (частиц) и “отрицательных" (античастищ) энергий гамильтониана Дирака-Паули для нейтрального фермиона в центрально-симметричном электрическом поле принципиально отличаются от аналогичных спектров гамильтониана Дирака для заряженного фермиона в центральном электрическом поле. В частности, спектры энергий уравнения Дирака-Паули симметричны относительно уровня Ферми $E_{\mathrm{F}}=0$, причем все дискретные уровни связанных состояний лежат выше $m$, так что минимальная ширина "шели" между дискретными уровнями, принадлежашими положительно- и отрицательно-частотным состояниям, равна $2 m$. Спектры энергий уравнения Дирака для заряженного фермиона не симметричны относительно уровня $E_{\mathrm{F}}=0$.

В самом деле, спектр “положительных" энергий уравнения Дирака для электрона в кулоновском поле положительно заряженного ядра дискретен в области $m>\mathcal{E} \geqslant 0$ и непрерывен в области $\mathcal{E}>m$, но в том же поле ядра в области $0>\mathcal{E}>-m$ нет ни одного отрицательно-частотного (позитронного) состояния. При этом минимальная ширина “щели” между уровнями, принадлежашими положительно- и отрицательно-частотным состояниям, меньше $2 m$.

\section{5. НУЛЕВЫЕ МОДЫ}

Найденные выше решения и спектр энергий уравнения Дирака-Паули для нейтрального фермиона с АММ в присутствии внешних электрических полей допускают переход к безмассовому пределу $m=0$. Заметим, что с точки зрения вторично квантованной теории в такой модели элементарный фермион имеет “затравочную” массу $m=0$, но только по отношению к вакууму в отсутствие внешнего электрического поля; по отношению же к вакууму в присутствии внешнего электрического поля фермион имеет не- 
нулевую массу за счет взаимодействия АММ с внешним полем (см. формулу (44)). Интересно, что в обсуждаемой модели с нулевой “затравочной” массой $m=0$ сушествуют фермионные состояния с нулевой энергией. Решения с нулевой энергией называют нулевыми модами.

Теоретически фермионные состояния с нулевой энергией впервые были обнаружены Джакивом и Ребби [23] в квантовой теории размерности $1+1$, описываюшей безмассовое дираковское поле, взаимодействуюшее с внешним топологическим солитонным полем (кинком). Эта теория симметрична относительно преобразования зарядового сопряжения (т.е. зарядово-самосопряжена). В этой модели существование невырожденного нормируемого зарядово-самосопряженного решения уравнения Дирака, описываюшего фермионное состояние с нулевой энергией, является определяющим и приводит к тому, что у безмассового дираковского поля появляются состояния с полуцелым фермионным числом $\pm 1 / 2$; появление таких состояний оказывается тесно связанным со спектральной симметрией модели. Джакив [24] также показал, что вакуум солитонного сектора в модели безмассовых фермионов, взаимодействующих с топологическим солитонным полем, и известная аксиальная аномалия, обнаруженная в [25], [26], могут быть интерпретированы как явления, возникаюшие из-за перестройки ветвей спектров положительных и отрицательных энергетических состояний соответствующих моделей. Отметим, что состояния с электронным числом $1 / 2$ открыты в полимерах (в полиацетилене) [27].

Дискретные уровни энергии связанных состояний (кинематически) безмассовых нейтральных фермионов с АММ в центрально-симметричных электрических полях определяются формулами (44), в которых нужно положить $m=0$. Тогда, низший уровень энергии, принадлежаший положительно-частотным состояниям с $s=-1$, есть $\mathcal{E}_{0 l s}=0$. За исключением его, для каждого ненулевого собственного значения $\mathcal{E}_{n l s}$ имеем пару собственных значений гамильтониана Дирака-Паули в соответствии с приведенными выше соображениями, основанными на зарядовом сопряжении. Тот факт, что непрерывный спектр начинается с $\mathcal{E}=\mu a$, согласуется с тем обстоятельством, что фермион (с $m=0)$ имеет массу $\mu a$.

При $\mathcal{E}=0$ и $m=0$ функции $f_{s}$ и $g_{s}$ в системе $(30)$ оказьваются независимыми друг от друга и их можно также найти прямо из (30); эти функции, конечно, можно получить из найденных выше решений, полагая в них $\mathcal{E}=0$ и $m=0$. Найдем изолированные решения с нулевой энергией уравнения Дирака-Паули во внешних центрально-симметричных электрических полях для обшего случая $E(r)=d V(r) / d r$. Вьше было показано, что низший уровень энергии связанного состояния массивного нейтрального фермиона есть $\mathcal{E}=m=0$. Для этого случая решение системы (30) легко находится в квадратурах и имеет вид

$$
\begin{aligned}
& f_{s}=A r^{-s(l+1)} \exp \left[-\mu \int d V(r)\right], \\
& g_{s}=B r^{s(l+1)} \exp \left[\mu \int d V(r)\right],
\end{aligned}
$$

а кооэффициенты $A$ и $B$ определяются из условия нормировки и ограниченности решений. 
Найдем сначала нулевые моды в явном виде в рассмотренных выше электрических полях. Нормированные ограниченные при $r=0$ решения в радиально-постоянном поле $E=a$ имеют вид

$$
\begin{gathered}
f_{-1}(\mu a>0)=A r^{l+1} e^{-\mu a r}, \quad f_{1}=g_{s}=0, \quad A=\frac{(2 \mu a)^{l+\frac{3}{2}}}{\sqrt{2(l+1) !}} \\
g_{1}(\mu a<0)=f_{-1}(\mu a>0), \quad g_{-1}=f_{s}=0 .
\end{gathered}
$$

Нормированные ограниченные при $r=0$ нулевые моды в поле $E=a r$ нетрудно получить в виде

$$
\begin{gathered}
f_{-1}(\mu a>0)=A r^{l+1} e^{-\frac{\mu a r^{2}}{2}}, \quad f_{1}=g_{s}=0, \quad A=\frac{2^{\frac{3}{4}}(\mu a)^{\frac{l}{2}+\frac{3}{4}}}{\sqrt{\Gamma\left(l+\frac{3}{2}\right)}} \\
g_{1}(\mu a<0)=f_{-1}(\mu a>0), \quad g_{-1}=f_{s}=0
\end{gathered}
$$

где $Г(z)$ - гамма-функция.

Найдем также решения для случая кулоновского поля $E(r)=-a / r^{2}, V(r)=a / r$, $a>0$. В этом поле нормированные ограниченные при $r=0$ решения с нулевой энергией можно найти в виде

$$
\begin{gathered}
f_{1}(\mu a>0)=A r^{-l-1} e^{-\frac{\mu a}{r}}, \quad f_{-1}=g_{s}=0, \quad A=\frac{(\mu a)^{l+\frac{1}{2}}}{\sqrt{2 l !}}, \\
g_{-1}(\mu a<0)=f_{1}(\mu a>0), \quad g_{1}=f_{s}=0 .
\end{gathered}
$$

Решения (46)-(48) являются изолированными.

Мы видим, что если "масса" нейтрального фермиона полностью генерируется электрическим полем, то сушествует счетное бесчисленное множество пар изолированных зарядово-сопряженных решений уравнения Дирака-Паули для нейтрального фермиона с АММ во внешних центрально-симметричных электрических полях. Найденные нами пары решений с нулевой энергией вырожденны и не являются зарядово-самосопряженными решениями (при преобразовании зарядового сопряжения получается решение с другим знаком проекции спина фермиона), поэтому полуцелые фермионные заряды вакуума не будут фигурировать во вторично квантованной теории.

Итак, дискретный спектр энергий массивного нейтрального фермиона с АММ в аксиально-симметричном магнитном поле (6) лежит в области $\mathcal{E} \leqslant m$ (при $p=0$ ), а в центрально-симметричных электрических полях - в области $\mathcal{E} \geqslant m$. Для электрона имеет место обратная картина: квазидискретньй спектр энергий электрона в магнитном поле лежит в области $\mathcal{E} \geqslant m$, а в центрально-симметричных электрических полях - в области $\mathcal{E} \leqslant m$. Дискретные уровни энергий нейтрального фермиона с АММ в аксиально-симметричном магнитном поле (6) (при $p=0$ ), принадлежащие областям $m \geqslant \mathcal{E}>0$ и $0>\mathcal{E} \geqslant-m$, симметричны относительно уровня $E_{\mathrm{F}}=0$ лишь при $b=0$. Поэтому пересечение уровней энергии из этих областей может иметь место в точке 
$\mathcal{E}=0$ при $b=0$, если $\mu a=1 / 2$. Низший уровень энергии $(\mathcal{E}=m)$ массивного нейтрального фермиона с АММ в центрально-симметричных электрических полях вырожден с бесконечной кратностью, а спин фермиона ориентирован против сохраняюшегося вектора момента $\mathbf{J}$.

Интересен вопрос о поведении квантово-механической системы, содержашей большое число нейтральных фермионов с АММ, в таких полях. Чтобы снять громадное вырождение в системе, необходимо учесть взаимодействие между фермионами, которое, очевидно, может быть обусловлено прежде всего магнитным взаимодействием спиновых магнитных дипольных моментов фермионов. Для системы, содержащей два фермиона, это взаимодействие должно приводить к связи спинов частиц и, следовательно, имеет вид

$$
V=J \mathbf{S}_{1} \cdot \mathbf{S}_{2}
$$

Известно [28], что член вида (49) может появиться и за счет обменного взаимодействия, явно не зависяшего от спина, при учете приншипа Паули. Это взаимодействие, определяемое обменным интегралом $J$, зависит от того, насколько сильно перекрываются волновые функции двух фермионов.

Квантово-механическая система большого числа массивных нейтральных фермионов с АММ в сферически-симметричных электрических полях в основном состоянии при учете взаимодействия вида (49) с $J<0$ в пределе бесконечного числа частиц имеет сходство с гейзенберговским ферромагнетиком. Гейзенберговский ферромагнетик, как известно [29], дает пример модели, в которой спонтанное нарушение симметрии приводит к возникновению спонтанной намагниченности, что представляется чрезвычайно интересным, если мы вспомним, что центральные области нейтронных звезд, как предполагается [30], характеризуются сверхвысокими плотностями нейтронов и протонов (протоны могут создать центрально-симметричное электрическое поле) и "вмороженным" (в звезду) магнитным полем. Впрочем, вопрос о возможности применения рассмотренных здесь квантово-механических систем для моделирования физических процессов, которые, по-видимому, могут протекать в центральной области нейтронной звезды, должен быть изучен отдельно.

Благодарности. Мне приятно поблагодарить А. В. Борисова, О.Ф. Дорофеева и Б. А. Лысова за плодотворные дискуссии.

\section{Список литературы}

[1] W. Pauli. Rev. Mod. Phys. 1941. V. 13. P. 203.

[2] Л. Д. Ландау. ЖЭТФ. 1957. Т. 32. С. 405.

[3] J. Schwinger. Particles, Sources, and Fields. Don Mills, Ontario: Addison-Wesley, 1970.

[4] И. М. Тернов, В. Г. Багров, Ю. И. Клименко. Изв. вузов. Физика. 1968. № 2. С. 50.

[5] Ю. И. Клименко, В. В. Кулии, А. И. Худомясов. Изв. вузов. Физика. 1974. № 10. С. 142.

[6] П. М. Лавров. Изв. вузов. Физика. 1977. № 12. С. 68.

[7] И. М. Тернов, В. Г. Багров. ЯФ. 1966. Т. 4. С. 797; ДАН СССР. 1966. Т. 168. С. 1298.

[8] Ю.И. Клименко. ЯФ. 1978. Т. 27. С. 1677.

[9] В. Г. Багров, В. Е. Степанов. Изв. вузов. Физика. 1967. № 1. С. 142.

[10] И. М. Тернов, В. Г. Багров, П. В. Бозриков. Изв. вузов. Физика. 1971. № 11. С. 39. 
[11] Y. Aharonov, A. Casher. Phys. Rev. Lett. 1984. V. 53. P. 319; A.S. Goldhaber. Phys. Rev. Lett. 1989. V. 62. P. 482.

[12] C. Cimmino, G.I. Opat, A. G. Klein, H. Kaiser, S. A. Werner, M. Arif, R. Clothier. Phys. Rev. Lett. 1989. V. 63. P. 380.

[13] J. Schwinger. Phys. Rev. 1948. V. 73. P. 407.

[14] Н. Н. Боголюбов, Д. В. Ширков. Квантовые поля. М.: Наука, 1980.

[15] В. Б. Берестецкий, Е. М. Лифиии, Л. П. Питаевский. Квантовая электродинамика. М.: Наука, 1980.

[16] V.R. Khalilov, C. L. Ho. Mod. Phys. Lett. A. 1998. V. 13. P. 615; C. L. Ho, V. R. Khalilov. Phys. Rev. A. 2000. V. 61. P. 032104.

[17] П. А. М. Дирак. Принципы квантовой механики. М.: Наука, 1979.

[18] В. Г. Багров, Д. М. Гитман, И. М. Тернов, В.Р. Халилов, В. Н. Шаповалов. Точные решения релятивистских волновых уравнений. Новосибирск: Наука, 1982.

[19] Q.-G. Lin. Phys. Rev. A. 2000. V. 61. P. 022101.

[20] К. Ичиксон, Ж.-Б. Зюбер. Квантовая теория поля. Т. 1. М.: Мир, 1984.

[21] И. С. Градитейн, И. М. Рыжик. Таблицы интегралов, сумм, рядов и произведений. М.: ГИФМЛ, 1963.

[22] Н. Я. Виленкин. Специальные функции и теория представлений групп. М.: Наука, 1965.

[23] R. Jackiw, C. Rebbi. Phys. Rev. D. 1976. V. 13. P. 3398.

[24] R. Jackiw. Effect of Dirac's negative energy sea on quantum numbers. hep-th/9903255.

[25] S. Adler. Phys. Rev. 1969. V. 177. P. 2496.

[26] J.S. Bell, R. Jackiw. Nuovo Cimento A. 1969. V. 60. P. 47.

[27] W. P. Su, J. R. Schrieffer, A. J. Heeger. Phys. Rev. Lett. 1979. V. 2. P. 1698.

[28] Р. Фейнман. Статистическая механика. М.: Мир, 1975.

[29] К. Ициксон, ЖК.-Б. Зюбер. Квантовая теория поля. Т. 2. М.: Мир, 1984.

[30] С. Вейнберг. Гравитация и космология. М.: Мир, 1975. 